\title{
Altered Functional Connectivity of Cognitive-Related Cerebellar Subregions in Well-Recovered Stroke Patients
}

\author{
Wei Li, ${ }^{1}$ Tong Han, ${ }^{1}$ Wen Qin, ${ }^{1}$ Jing Zhang, ${ }^{1}$ Huaigui Liu, ${ }^{1}$ Ying Li, ${ }^{1}$ Liangliang Meng, \\ Xunming Ji, ${ }^{2}$ and Chunshui Y ${ }^{1}$ \\ ${ }^{1}$ Department of Radiology and Tianjin Key Laboratory of Functional Imaging, Tianjin Medical University General Hospital, \\ Tianjin 300052, China \\ ${ }^{2}$ Cerebrovascular Diseases Research Institute, Xuanwu Hospital of Capital Medical University, Beijing 100053, China
}

Correspondence should be addressed to Chunshui Yu; chunshuiyu@tijmu.edu.cn

Received 9 March 2013; Revised 3 June 2013; Accepted 4 June 2013

Academic Editor: Bruno Poucet

Copyright (C) 2013 Wei Li et al. This is an open access article distributed under the Creative Commons Attribution License, which permits unrestricted use, distribution, and reproduction in any medium, provided the original work is properly cited.

\begin{abstract}
The cerebellum contains several cognitive-related subregions that are involved in different functional networks. The cerebellar crus II is correlated with the frontoparietal network (FPN), whereas the cerebellar IX is associated with the default-mode network $(\mathrm{DMN})$. These two networks are anticorrelated and cooperatively implicated in cognitive control, which may facilitate the motor recovery in stroke patients. In the present study, we aimed to investigate the resting-state functional connectivity (rsFC) changes in 25 subcortical ischemic stroke patients with well-recovered global motor function. Consistent with previous studies, the crus II was correlated with the FPN, including the dorsolateral prefrontal cortex (DLPFC) and posterior parietal cortex, and the cerebellar IX was correlated with the DMN, including the posterior cingulate cortex/precuneus (PCC/Pcu), medial prefrontal cortex (MPFC), DLPFC, lateral parietal cortices, and anterior temporal cortices. No significantly increased rsFCs of these cerebellar subregions were found in stroke patients, suggesting that the rsFCs of the cognitive-related cerebellar subregions are not the critical factors contributing to the recovery of motor function in stroke patients. The finding of the disconnection in the cerebellar-related cognitive control networks may possibly explain the deficits in cognitive control function even in stroke patients with well-recovered global motor function.
\end{abstract}

\section{Introduction}

Ischemic stroke is one of the leading causes of motor disability in adults, while most patients experience a certain degree of recovery of motor function. The mechanisms of motor recovery after stroke have been extensively investigated especially using the neuroimaging techniques [1-6]. However, the contribution of cerebellum to motor recovery after stroke is a subject of much debate. The contralesional cerebellum is a part of the affected motor network and has reciprocal connections with the ipsilesional sensorimotor cortex. In subcortical stroke, contralesional cerebellar hypometabolism [7-9] and atrophy [10] are reported and have been ascribed to the damage of anatomical connections by lesions [11, 12]. Initially the affected cerebellum has been shown to exhibit greater activation during performing a motor [13] or a tactile exploration task [14] with the affected hand. Subsequently, this activation decreased gradually and this was correlated with functional recovery [15]. In a longitudinal analysis of the executive motor network, the betweenness centrality (a measure evaluating the importance of a node in brain network) of the affected cerebellum was negatively correlated with the recovery of motor function [16].

Beyond the motor function, the cerebellum is also critical for cognitive control [17-23]. Sang et al. [24] have showed detailed resting-state functional connectivity ( $\mathrm{rsFC}$ ) patterns of cerebellar subregions in healthy subjects. In that study [24], the cerebellar crus II was typically connected with the frontoparietal network (FPN) which is associated with cognitive control and the cerebellar IX was mainly connected with the default-mode network (DMN), which is deactivated during cognitively demanding tasks. Other studies have also identified that cerebellum IX contributes to the DMN [24, 25], cerebellar VII (including crus II) contributes to higherlevel processes $[21,24,26]$, and they were activated during cognitive paradigm $[27,28]$. 


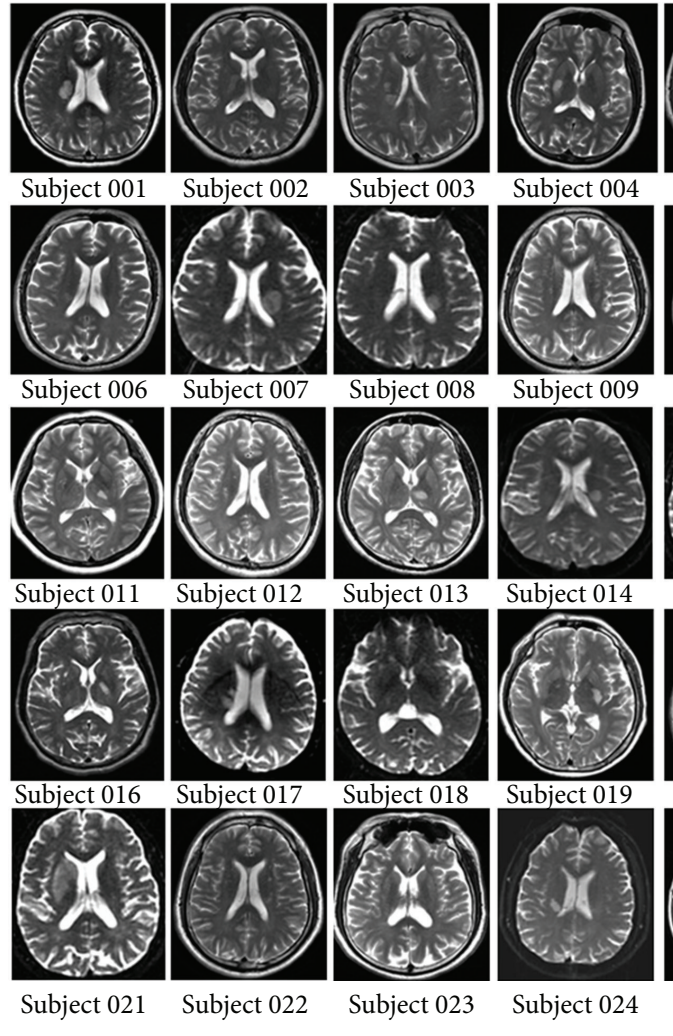

(a)

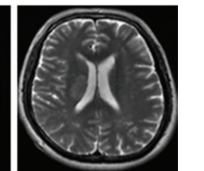

Subject 005

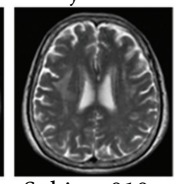

Subject 010

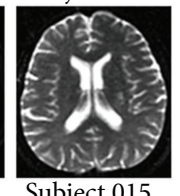

Subject 015

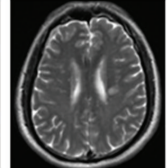

Subject 020

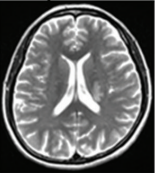

Subject 025
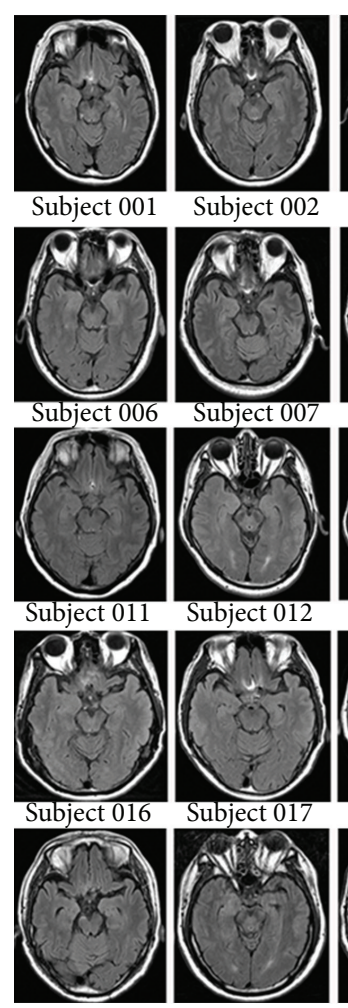

Subject 021

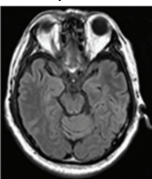

Subject 007
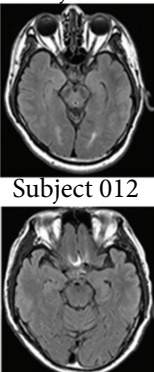

Subject 017

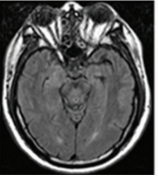

Subject 022

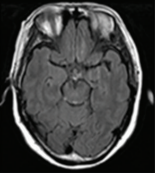

Subject 003
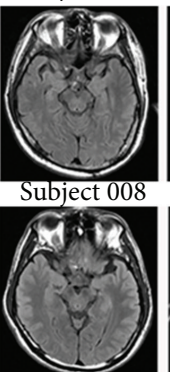

Subject 013

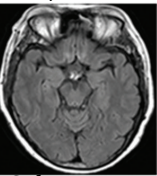

Subject 018

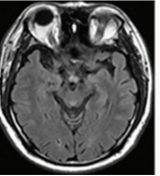

Subject 023

(b)

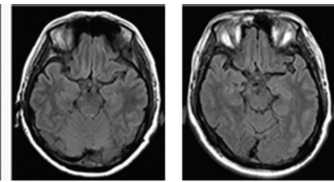

Subject 004 Subject 005
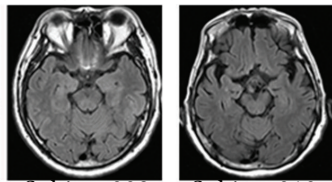

Subject 009 Subject 010

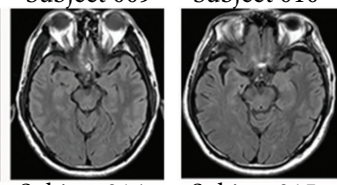

Subject 014 Subject 015

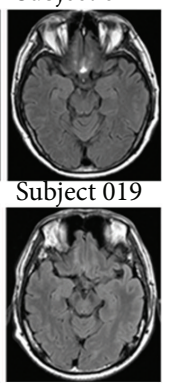

Subject 024

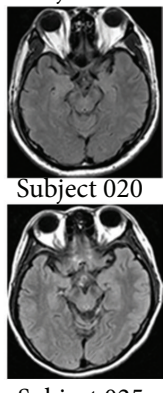

Subject 025

Figure 1: (a) Lesion locations of stroke patients on axial T2-weighted images acquired at the stroke onset when stroke patients were first enrolled in our hospital. (b) The morphology and signals of the brainstem of every stroke patient.

Although the recovery of motor function in stroke patients is associated with the reconstruction of the motor network, it is suggested that the recovery of motor function is a process of relearning the motor skills [29-31]. The cerebellar subregions (crus II and lobule IX), as important nodes in relearning the motor skills, may contribute to motor recovery after stroke. If the hypothesis is correct, we predict that the rsFCs between the cerebellar subregions (crus II and lobule IX) and the cerebral regions of the FPN and DMN will increase in stroke patients with good recovery in global motor function; in contrast, the decreased rsFCs may indicate functional impairment of the two cerebellum-related cognitive control networks (FPN and DMN) in these stroke patients. In the present study, we aimed to investigate the rsFC changes of these two cognitive-related cerebellar subregions in 25 well-recovered subcortical ischemic stroke patients to clarify their roles in motor recovery after stroke.

\section{Method and Materials}

2.1. Subjects. The experiment was approved by the Ethical Committee of Tianjin Medical University General Hospital and written informed consent was obtained from each subject before the study. Inclusion criteria were as follows: (1) firstonset stroke; (2) manifested motor deficit at stroke onset; (3) unilateral single lesion involving the internal capsule and neighboring regions; (4) being right-handed; (5) 6 months or more since the time of infarct; (6) with good recovery of motor function, the upper extremity Fugl-Meyer test (UE_FMT) of $>60$, and the whole extremity Fugl-Meyer test (WE_FMT) of $>90$; (7) compliance to carry out the experiment. Exclusion criteria were as follows: (1) recurrent stroke after first onset; (2) with any other brain abnormalities other than the infarct lesion, either history of neurological or psychiatric disorders, or did any of them experience subsequent symptomatic stroke; (3) severe white matter hyperintensity revealed by T2W images; (4) history of drug dependency or psychiatric disorders; (5) conscious disturbance or uncooperative; (6) head motion parameters of fMRI data satisfied a maximum displacement of $<2 \mathrm{~mm}$ or a maximum rotation of $<2.0^{\circ}$. According to the criteria, 25 patients ( 7 females; mean age: 56.2 years; range: $42-72$ years) were finally included in this study (Figure 1, Table 1). Twenty-two age-matched healthy controls (11 females; mean age: 57.2; range: $47-74$ years) were also recruited as controls who reported no history of psychiatric or neurological problems and the head motion parameters of fMRI data satisfied a maximum displacement of $<2 \mathrm{~mm}$ or a maximum rotation of $<2.0^{\circ}$.

2.2. Data Acquisition. Sagittal 3D T1-weighted images were acquired by a brain volume (BRAVO) sequence with the following parameters: repetition time (TR)/echo time (TE) $=8.1 / 3.1 \mathrm{~ms}$; field of view $(\mathrm{FOV})=256 \times 256 \mathrm{~mm}^{2}$; matrix $=256 \times 256$; slice thickness $=1.0 \mathrm{~mm}$, no gap; 176 slices. The 
TABLE 1: Clinical information of stroke patients.

\begin{tabular}{|c|c|c|c|c|c|c|c|}
\hline \multirow{2}{*}{ ID } & \multirow{2}{*}{ Sex } & \multirow{2}{*}{ Age (year) } & \multirow{2}{*}{ Duration (month) } & \multirow{2}{*}{ Lesion location } & \multirow{2}{*}{ MMSE } & \multicolumn{2}{|c|}{ FMT } \\
\hline & & & & & & UE & WE \\
\hline BG001 & $\mathrm{F}$ & 65 & 63 & Right PLIC, CR & 29 & 62 & 94 \\
\hline BG002 & M & 62 & 41 & Right PLIC, GIC & 27 & 66 & 100 \\
\hline BG003 & $\mathrm{F}$ & 63 & 48 & Right CR, PLIC, LN & 30 & 65 & 98 \\
\hline BG004 & $\mathrm{F}$ & 52 & 64 & Right CR, PLIC, LN & 30 & 65 & 99 \\
\hline BG005 & M & 53 & 37 & Right CR, PLIC, LN & 1 & 62 & 95 \\
\hline BG006 & M & 65 & 37 & Right CR & 28 & 66 & 98 \\
\hline BG007 & M & 59 & 41 & Left CR, PLIC, LN & 29 & 64 & 98 \\
\hline BG008 & M & 49 & 40 & Left CR, PLIC, LN & 28 & 65 & 98 \\
\hline BG009 & M & 60 & 30 & Left CR & 26 & 66 & 100 \\
\hline BG010 & $\mathrm{F}$ & 72 & 41 & Right CR, PLIC, LN & 26 & 65 & 98 \\
\hline BG011 & $\mathrm{F}$ & 55 & 24 & Left Th & 26 & 66 & 100 \\
\hline BG012 & M & 49 & 24 & Right CR, LN & 30 & 66 & 100 \\
\hline BG013 & M & 42 & 24 & Left Th & 28 & 66 & 100 \\
\hline BG014 & M & 50 & 18 & Left CR, PLIC & 27 & 66 & 100 \\
\hline BG015 & M & 52 & 22 & Left CR, PLIC & 29 & 66 & 100 \\
\hline BG016 & M & 58 & 52 & Left PLIC & 29 & 66 & 100 \\
\hline BG017 & M & 65 & 20 & Right CR, PLIC, LN & 28 & 65 & 99 \\
\hline BG018 & $\mathrm{F}$ & 63 & 14 & Right Th & 28 & 66 & 100 \\
\hline BG019 & M & 55 & 11 & Left PLIC, LN & 30 & 66 & 100 \\
\hline BG020 & M & 47 & 13 & Left CR & / & 66 & 100 \\
\hline BG021 & M & 58 & 14 & Right CR, Cau, ALIC & l & 66 & 100 \\
\hline BG022 & M & 63 & 13 & Left CR, PLIC & 27 & 66 & 100 \\
\hline BG023 & M & 45 & 11 & Right CR & 30 & 66 & 100 \\
\hline BG024 & M & 49 & 13 & Right CR, PLIC & 30 & 64 & 96 \\
\hline BG025 & $\mathrm{F}$ & 53 & 11 & Left CR, PLIC, LN & 28 & 66 & 99 \\
\hline
\end{tabular}

ALIC: anterior limb of internal capsule; Cau: caudate; CR: corona radiate; GIC: genu of internal capsule; LN: lenticular nucleus; PLIC: posterior limb of internal capsule; Th: thalamus.

resting-state fMRI data of all the subjects were obtained using a gradient-echo single-shot echo-planar imaging sequence (GRE-SS-EPI) with the following parameters: TR/TE = $2000 / 30 \mathrm{~ms}$; slice thickness $=3 \mathrm{~mm}, 1 \mathrm{~mm}$ gap; matrix $=64 \times$ 64 ; FOV $=240 \times 240 \mathrm{~mm}^{2} ; 38$ transverse slices; 180 volumes. During fMRI scans, all subjects were instructed to keep their eyes closed, to stay as motionless as possible, to think of nothing in particular, and not to fall asleep.

2.3. Data Preprocessing. Before the data preprocessing, we have flipped the imaging data from left to right about the midsagittal line for patients with lesions on the left hemisphere. For all patients, the right side was the ipsilesional (affected) hemisphere while the left side was the contralesional (unaffected) hemisphere. The resting-state fMRI data were preprocessed using Statistical Parametric Mapping (SPM8, http://www.fil.ion.ucl.ac.uk/spm/). The first 10 volumes from each subject were discarded to allow the signal to reach equilibrium and the participants to adapt to the scanning noise. The remaining 170 volumes were corrected for acquisition time delay between slices. Then, head motion parameters were estimated; none of the 47 subjects had a maximum displacement of $>2 \mathrm{~mm}$ or a maximum rotation of $>2.0^{\circ}$. In this step, the framewise displacement (FD) was also calculated to characterize the instantaneous head motion of each volume. The remaining data set was then normalized into the Montreal Neurological Institute (MNI) EPI template and resampled into $3 \times 3$ $\times 3 \mathrm{~mm}^{3}$ voxels. Thereafter, some nuisance variables were regressed from the BOLD data, including the averaged signals of the ventricular, white matter, the whole brain, six head realignment parameters obtained by rigid body head motion correction, and the temporal derivatives of each of these realignment parameters. Because recent studies found that signal spike caused by motion also significantly contaminates the final resting-state fMRI results even after regressing out the realignment parameters, in this study, we also removed the spike volumes by generating an individual vector when the FD of specific volume exceeded 0.5. Next, a band-pass frequency filter $(0.01-0.08 \mathrm{~Hz})$ was applied to reduce lowfrequency drift and high-frequency noise. Finally, the filtered BOLD images were spatially smoothed with an isotropic Gaussian kernel of FWHM $=8 \mathrm{~mm}$.

2.4. Definition of Cerebellar ROIs. The bilateral cerebellum subregions of crura II and IX were extracted from the Probabilistic cerebellar atlas [32] with a threshold of 50\% minimum probability (Figure 2). 


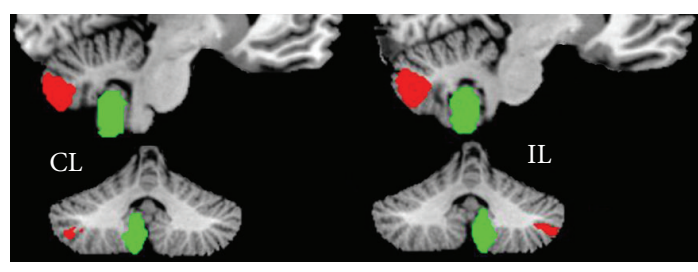

\section{Crus II IX}

FIGURE 2: The bilateral seed regions of the cerebellar subregions of crura II and IX extracted from the probabilistic cerebellar atlas with a threshold of $50 \%$ minimum probability.

2.5. The rsFC Analysis of Cerebellar Crura II and IX. For each individual dataset, Pearson's correlation coefficients between the mean time series of each seed region and that of each voxel of the whole brain were computed and converted to $z$ values using Fisher's $r$-to- $z$ transformation to improve the normality. Then, individuals' $z$-values were entered into a random-effect one-sample $t$-test in a voxel-wise manner to identify brain regions that showed significant positive correlations with the seed region. A corrected threshold of $P<0.05$ was derived from a combined threshold of $P<$ 0.01 for each voxel and a cluster size $>65$ voxels which was determined by the AlphaSim program in the AFNI software (parameters: single voxel $P=0.01,5000$ simulations, FWHM $=8 \mathrm{~mm}$, cluster connection radius $=5 \mathrm{~mm}$, with gray matter mask, http://afni.nimh.nih.gov/).

\subsection{Intergroup Comparisons of the rsFCs of the Cerebellar} Crura II and IX. A two-sample $t$-test was performed to quantitatively compare differences in rsFCs of the two cerebellar subregions between controls and patients with age and gender being treated as covariates of no interest. A corrected threshold of $P<0.05$ was derived from a combined threshold of $P<0.01$ for each voxel and a cluster size $>31$ voxels which was determined by the AlphaSim program in the AFNI software (parameters: single voxel $P=0.01,5000$ simulations, FWHM $=8 \mathrm{~mm}$, cluster connection radius = $5 \mathrm{~mm}$, with a mask of gray matter regions showing positive rsFC with each cerebellar ROI, http://afni.nimh.nih.gov/).

2.7. The Analysis of Subgroup Stroke Patients Group. To reduce the influence of the inconsistency of lesion location on our results, we selected 11 stroke patients with lesions involving the posterior limb of internal capsule, through which the corticospinal tracts pass. Using the same method, we run the one-sample $t$-test in stroke subgroup patients and the twosample $t$-test between stroke subgroup patients and healthy controls.

\section{Results}

3.1. Demographic and Clinical Information. Twenty-five subcortical stroke patients with well-recovered motor function and twenty-two normal controls were recruited in the present study. The clinical and demographic data of the stroke patients are listed in Table 1. The lesion location of each patient was demonstrated on axial T2W images, which were acquired at the stroke onset when stroke patients were first enrolled in our hospital (Figure 1(a)). Compared with the healthy controls, stroke patients did not show any significant differences in both age (two-sample $t$-test: $t_{45}=-0.50, P=$ 0.62 ) and gender (chi-square test: $\chi_{1}^{2}=2.40, P=0.12$ ). As shown in Table 1, the duration from stroke onset to MRI scanning ranged from 11 months to 64 months (mean value: $29.0 \pm$ 16.4 months). The ischemic lesions involved the internal capsule regions and surrounding structures, including the internal capsule, lentiform nucleus, caudate nucleus, corona radiate, and thalamus; thirteen out of the 25 stroke patients had infarct lesion in the right hemisphere and 12 in the left hemisphere. Except for 3 patients that failed to measure the MMSE score, the remaining 22 patients showed relative normal cognitive status (MMSE ranged from 26 to 30 , mean $28.3 \pm 1.3$ ). The Fugl-Meyer test showed that both the motor functions of upper and lower extremities were well recovered (minimum 62 for upper extremities and 94 for all extremities).

3.2. Whole-Brain rsFC Patterns of the Cerebellar Crus II. We only focused on the positive rsFCs of each cerebellar subregion because the functional significance of the negative rsFC is a matter of debate that whether the negative connectivity is an artifact of the global signal regression $[33,34]$ or reflects dynamic, anticorrelated functional networks [35]. In healthy controls, the cerebellar crus II was mainly correlated with the contralateral FPN, including the dorsolateral prefrontal cortex (DLPFC) and posterior parietal cortex (see Figure 3(a) and Table 2). Stroke patients showed similar rsFC patterns of the cerebellar crus II but the strengths and spatial extent were slightly different from those of healthy controls (see Figure 3(b) and Table 2).

3.3. Whole-Brain rsFC Patterns of the Cerebellar IX. In the healthy controls, the cerebellum IX showed strong rsFCs with the DMN mainly including the posterior cingulate cortex/precuneus (PCC/Pcu), medial prefrontal cortex (MPFC), DLPFC, lateral parietal cortices, and anterior temporal cortices (see Figure 3(a) and Table 2). Stroke patients showed similar rsFC patterns of the cerebellar IX but the strengths and spatial extent were lower relative to those of healthy controls (see Figure 3(b) and Table 2).

\subsection{Altered rsFC Patterns of Cerebellar Crus II in Stroke} Patients. Compared with healthy controls, stroke patients showed decreased rsFC between the contralesional cerebellar crus II and the ipsilesional DLPFC and between the ipsilesional cerebellar crus II and the contralesional caudate nucleus (Figure 3(c) and Table 2). In contrast, there was no significant increase in rsFC between the cerebellar crus II and any of cortical or subcortical forebrain regions.

3.5. Altered rsFC Patterns of Cerebellar IX in Stroke Patients. Compared with healthy controls, stroke patients showed decreased rsFC between the contralesional cerebellar IX 


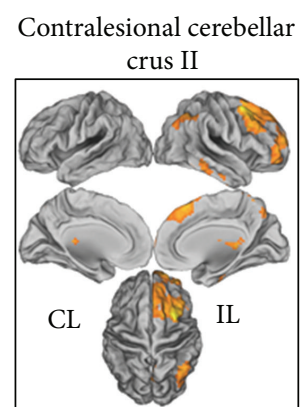

Ipsilesional cerebellar
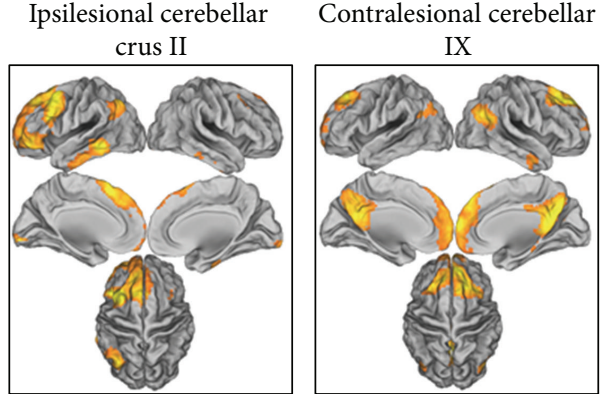

(a)
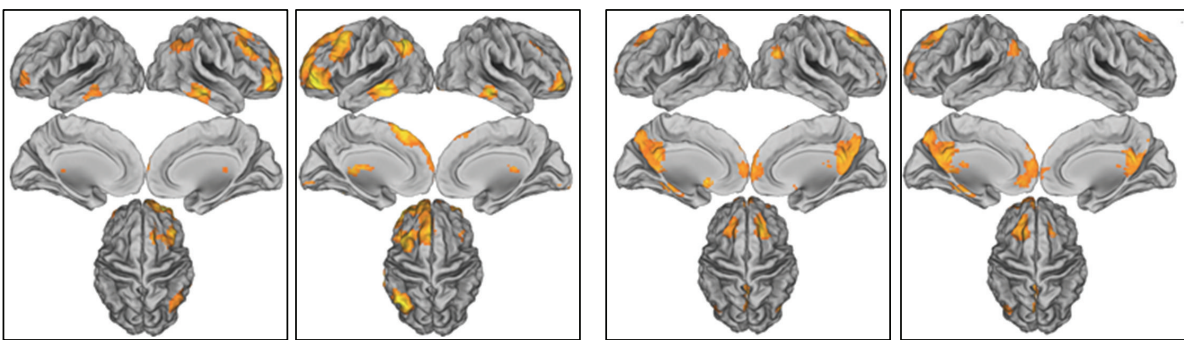

(b)
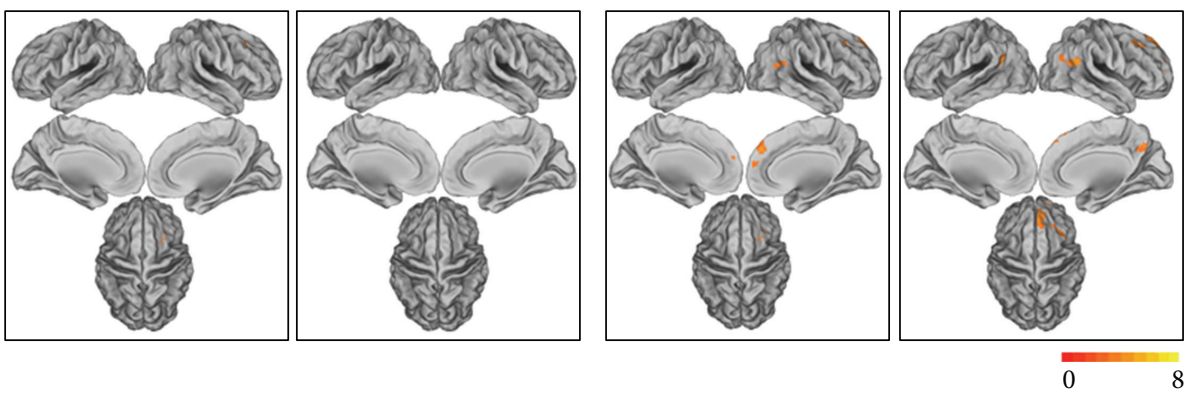

(c)

FIGURE 3: The rsFC patterns of the cerebellar subregions in healthy controls and stroke patients (total: $n=25$ ) and the rsFC differences between stroke patients and healthy controls $(P<0.05$ corrected). (a) The rsFC patterns of the cerebellar subregions in healthy controls; (b) the rsFC patterns of the cerebellar subregions in stroke patients; (c) the differences in the rsFCs between stroke patients and healthy controls.

and the ipsilesional MPFC, DLPFC, and lateral parietal cortices. Stroke patients also had decreased rsFC between the ipsilesional cerebellar IX and the ipsilesional PCC/Pcu and prefrontal areas and the bilateral lateral parietal cortices (Figure 3(c) and Table 2). In contrast, there was no significant increase in rsFC between the cerebellar IX and any of cortical or subcortical forebrain regions.

3.6. The Analysis of Subgroup of Stroke Patients. The results of the subgroup of stroke patients are shown in Figure 4. Both the rsFCs of stroke patients and intergroup differences were similar to those obtained from all stroke patients.

\section{Discussion}

4.1. The rsFC Patterns of the Cerebellar Crura II and IX. In the present study, we found that the cerebellar crus II was mainly correlated with the brain regions of the contralateral FPN, including the DLPFC and posterior parietal cortex, which is well consistent with previous findings on the FPN $[24,25$,
36-38]. A transneuronal tracing study also supports these findings by showing that crus II has reciprocal connections with the DLPFC [39]. We also found that the cerebellar lobule IX was mainly correlated with brain regions of the DMN, including the $\mathrm{PCC} / \mathrm{Pcu}, \mathrm{MPFC}$, lateral parietal cortices, DLPFC, and anterior temporal areas. This finding was in agreement with previous rsFC studies $[24,25,37,38]$.

4.2. The Relationship between the FPN and DMN. The FPN and DMN are the two most important cognitive-related functional networks in the human brain. The FPN is involved in cognitive control, such as working memory [40, 41] and executive control function [27], whereas the DMN is implicated in self-referential processing [42], conscious awareness [43], mind wandering [44], and retrieval and manipulation of episodic memories and semantic knowledge [45]. During the performance of cognitively demanding tasks, the FPN typically shows increases in activation, whereas the DMN shows decreases in activation $[46,47]$. During resting state, the FPN and DMN are anticorrelated with each other and 


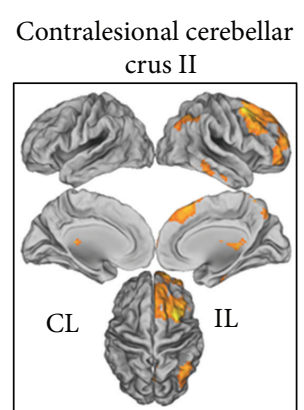

Ipsilesional cerebellar

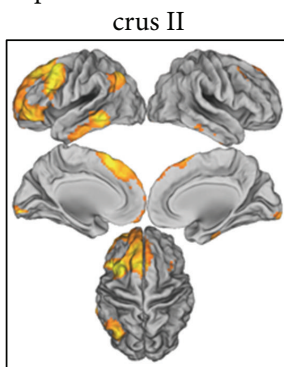

(a)
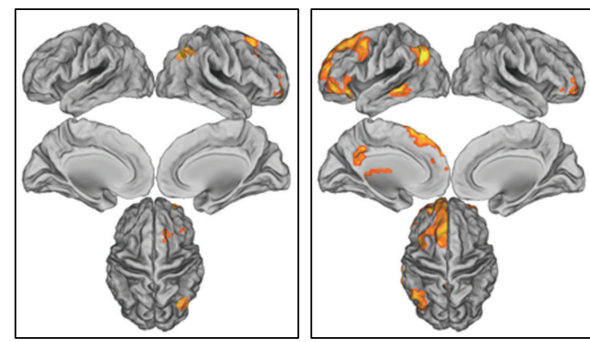

(b)
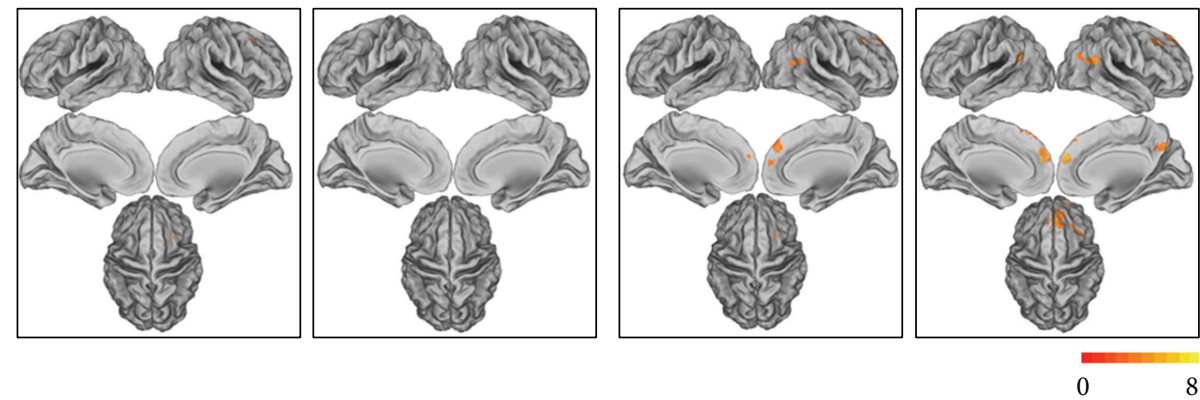

(c)

FIGURE 4: The rsFC patterns of the cerebellar subregions in healthy controls and stroke patients (subgroup: $n=11$ ) and the rsFC differences between stroke patients and healthy controls $(P<0.05$ corrected). (a) The rsFC patterns of the cerebellar subregions in healthy controls; (b) the rsFC patterns of the cerebellar subregions in stroke patients; (c) the differences in the rsFCs between stroke patients and healthy controls. the strength of this anticorrelation was associated with cognitive performance $[48,49]$. Consequently, we selected the cerebellar crura II and IX as subregions of interest to represent the cerebellar-related cognitive control regions.

\subsection{No Increased rsFC of the Cerebellar Crura II and IX} Subregions in Stroke Patients. The motor recovery of stroke patients is commonly thought of as a relearning process of the motor skill [29-31]. Various rehabilitation approaches have been used to improve skill reacquisition of the impaired arm [50] and enhanced clinical outcomes depend on both the training intensity and task specificity [51]. The cognitive condition or ability for the recovery of motor function has been extensively reported $[52,53]$ and cognitive strategy-based interventions have been shown to have beneficial effects on the recovery of motor function in stroke patients $[50,54]$. Thus the motor skill reacquisition may need the cognitive rehabilitation training. This inference is also supported by the finding that motor improvements of stroke patients were control system either during the spontaneous recovery or the
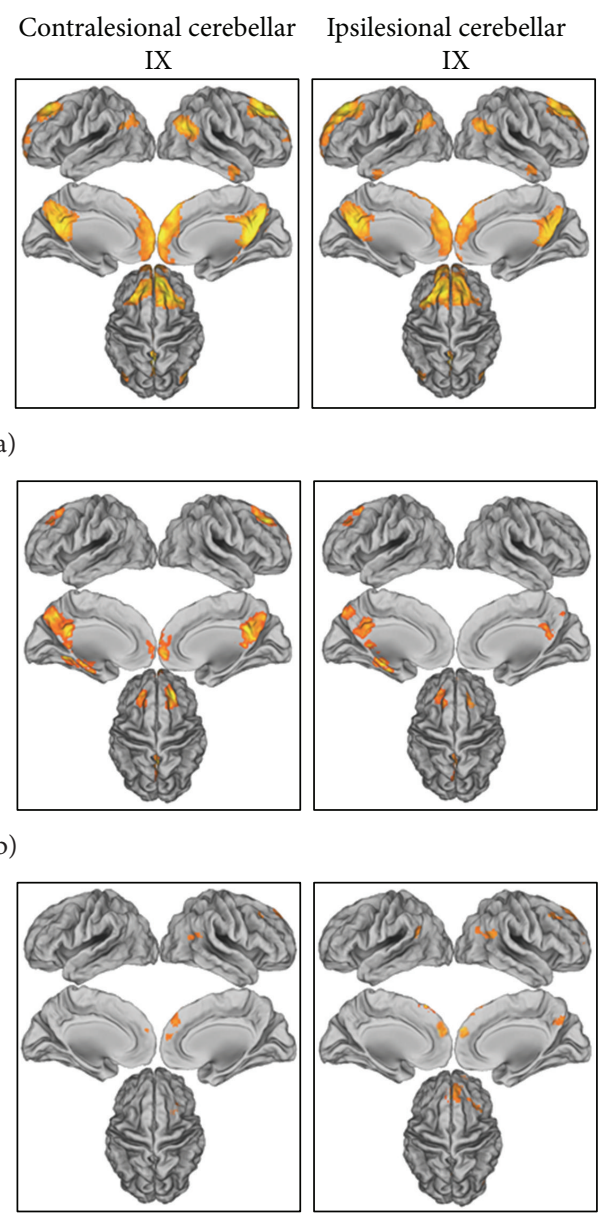

8
The rsFC patterns of the cerebellar subregions in healthy controls; (b)
differences in the rsFCs between stroke patients and healthy controls.

related to better memory, mental flexibility, and planning abilities [50].

In the present study, we aimed to test whether the rsFCs of the cognitive-related cerebellar subregions have beneficial effects on the recovery of motor function in stroke patients. Unexpectedly, we did not find any significantly increased rsFC of either the cerebellar crus II or the cerebellar lobule IX in stroke patients. These negative results suggest that the rsFCs of the cognitive-related cerebellar subregions do not contribute to the recovery of motor function in stroke patients. However, this negative finding does not exclude the possibility of the involvement of the cognitive-related brain regions in the motor recovery process because several studies have shown that cognitive-related brain regions such as the hippocampus were enlarged in chronic stroke patients [55] or after rehabilitation training [56].

4.4. Decreased rsFC of the Cerebellar Crura II and IX Subregions in Stroke Patients. In stroke patients, the cerebellar crus II showed decreased rsFCs with brain regions of the 
TABLE 2: The rsFCs of the cerebellar crura II and IX in both of the control and patient groups and significant group differences in these rsFCs between healthy controls and stroke patients.

\begin{tabular}{|c|c|c|c|c|c|}
\hline Seed regions & Connected regions & Group difference & Peak $t$ values & Peak coordinate MNI $(x, y, z)$ & Cluster size (voxels) \\
\hline \multirow{7}{*}{ CL_crus II } & IL_DLPFC & $*$ & 4.23 & $30,18,39$ & 74 \\
\hline & IL_LPC & & & & \\
\hline & IL_Pcu & & & & \\
\hline & IL_ITG & & & & \\
\hline & IL_MTG & & & & \\
\hline & CL_ITG & & & & \\
\hline & CL_DLPFC & & & & \\
\hline \multirow{9}{*}{ IL_crus II } & B_DLPFC & & & & \\
\hline & B_MPFC & & & & \\
\hline & IL_ITG & & & & \\
\hline & CL_LPC & & & & \\
\hline & CL_MTG/ITG & & & & \\
\hline & CL_PCC/Pcu & & & & \\
\hline & CL_Cal & & & & \\
\hline & CL_Cau & $*$ & 4.11 & $-12,6,15$ & 40 \\
\hline & CL_Th & & & & \\
\hline \multirow{10}{*}{ CL_IX } & B_PCC/Pcu & & & & \\
\hline & B_MCC & & & & \\
\hline & IL_MPFC & * & 3.42 & $6,48,33$ & 80 \\
\hline & IL_ACC & & & & \\
\hline & IL_MTG/MOG & & & & \\
\hline & IL_LPC & $*$ & 3.18 & $54,-57,18$ & 44 \\
\hline & IL_DLPFC & $*$ & 3.98 & $33,24,42$ & 38 \\
\hline & CL_DLPFC & & & & \\
\hline & CL_LPC & & & & \\
\hline & CL_MPFC & & & & \\
\hline \multirow{8}{*}{ IL_IX } & IL_MPFC & $*$ & 4.35 & $18,57,21$ & 241 \\
\hline & IL_LPC & $*$ & 4.14 & $54,-54,21$ & 103 \\
\hline & IL_DLPFC & $*$ & 3.97 & $36,24,45$ & 77 \\
\hline & IL_MTG & & & & \\
\hline & IL_PCC/Pcu & * & 3.30 & $15,-54,33$ & 51 \\
\hline & CL_ParaHP & & & & \\
\hline & CL_LPC & $*$ & 3.95 & $-51,-57,24$ & 66 \\
\hline & CL_PCC/Pcu & & & & \\
\hline
\end{tabular}

ACC: anterior cingulated cortex; Cal: calcarine; Cau: caudate; CL: contralesional hemisphere; DLPFC: dorsolateral prefrontal cortex; IL: ipsilesional hemisphere; ITG: inferior temporal gyrus; LPC: lateral parietal cortex; MCC: middle cingulate cortex; MOG: middle occipital gyrus; MPFC: the medial part of prefrontal cortex; MTG: middle temporal gyrus; ParaHP: parahippocampal; PCC: posterior cingulate cortex; Pcu: precuneus; Th: thalamus. The star ( $*$ ) represents that the rsFC shows significant group difference between stroke patients and healthy controls.

FPN and the cerebellar lobule IX exhibited decreased rsFC with brain regions of the DMN. These findings suggest that the decreased rsFCs of the cerebellar crus II and the cerebellar lobule IX are the hallmark in these stroke patients with well-recovered global motor function. It has been reported that subcortical stroke not only impaired the motor function but also impaired the cognitive function $[57,58]$ through damaging the white matter fibers that are important for cognitive processing. Volumetric studies have revealed the extensive atrophy in the bilateral frontal, parietal, and insular areas, especially the affected hemisphere $[1,58]$ which are important for cognitive function. The functional disconnection within the DMN has been reported in stroke patients and the disconnection was associated with the poststroke depression severity [59]. Additionally, the impaired FPN was also reported in stroke patients [60]. Beyond the previous findings of the involvement of the DMN and FPN, we provide evidence that the rsFCs between the cognitive-related cerebellar subregions (crus II of the FPN and lobule IX of the DMN) and cerebral regions of their corresponding networks (FPN and DMN) are also impaired in stroke patients even though they recovered well in global motor function. In addition, we found that most of cerebral regions showing impaired rsFCs with the two cerebellar subregions were located in the affected cerebral cortex, which further supports our inference that these decreased rsFCs 
may represent the impaired cognitive function in these stroke patients. However, the lack of specialized cognitive assessments impedes us to determine the relationship between these rsFC impairments and the behavioral performance in these stroke patients. Further studies should be performed to determine the relationship.

\section{Limitation}

A major weakness of this study is the inclusion of various groups of stroke, such as different lesion location and different clinical deficits. Although we reperformed the analyses in a subgroup of stroke patients with the involvement of the corticospinal tract and found similar results, further studies with larger sample size and more homogeneous clinical characteristics are needed to verify our findings. Another limitation of this study is the lack of evaluation of sensory and cognitive deficits, which should be done in future studies.

\section{Conclusion}

In the present study, we analyzed the alterations of the rsFCs of the cerebellar crura II and IX in stroke patients with well-recovered global motor function. We did not find any significantly increased rsFCs of these cerebellar subregions in stroke patients, suggesting that the rsFCs between cognitiverelated cerebellar subregions (crus II of the FPN and lobule IX of the DMN) and cerebral regions of their corresponding networks (FPN and DMN) do not experience plastic changes that may facilitate the motor recovery in subcortical stroke patients with good recovery of their global motor function. In contrast, stroke patients showed decreased rsFCs between these cerebellar subregions and their corresponding functional networks. These findings may represent functional disconnection within both the FPN and DMN even in well-recovered stroke patients, which may be associated the cognitive deficits in stroke patients.

\section{Conflict of Interests}

The authors declare that they have no conflict of interests.

\section{Authors' Contribution}

Wei Li and Tong Han contributed equally to the work.

\section{Acknowledgments}

This study was supported by the National Basic Research Program of China (973 program, 2011CB707804), the Natural Science Foundation of China (81271564), and the Natural Science Foundation of Tianjin (12JCZDJC23800).

\section{References}

[1] C. Calautti and J.-C. Baron, "Functional neuroimaging studies of motor recovery after stroke in adults: a review," Stroke, vol. 34, no. 6, pp. 1553-1566, 2003.
[2] C. Calautti, M. Naccarato, P. S. Jones et al., "The relationship between motor deficit and hemisphere activation balance after stroke: A 3T fMRI Study," NeuroImage, vol. 34, no. 1, pp. 322331, 2007.

[3] S. C. Cramer, G. Nelles, J. D. Schaechter, J. D. Kaplan, S. P. Finklestein, and B. R. Rosen, "A functional MRI study of three motor tasks in the evaluation of stroke recovery," Neurorehabilitation and Neural Repair, vol. 15, no. 1, pp. 1-8, 2001.

[4] S. Dechaumont-Palacin, P. Marque, X. De Boissezon et al., "Neural correlates of proprioceptive integration in the contralesional hemisphere of very impaired patients shortly after a subcortical stroke: An fMRI Study," Neurorehabilitation and Neural Repair, vol. 22, no. 2, pp. 154-165, 2008.

[5] I. Loubinoux, S. Dechaumont-Palacin, E. Castel-Lacanal et al., "Prognostic value of fMRI in recovery of hand function in subcortical stroke patients," Cerebral Cortex, vol. 17, no. 12, pp. 2980-2987, 2007.

[6] D. Tombari, I. Loubinoux, J. Pariente et al., "A Longitudinal fMRI Study: in recovering and then in clinically stable subcortical stroke patients," NeuroImage, vol. 23, no. 3, pp. 827-839, 2004.

[7] S. Pappata, B. Mazoyer, S. Tran Dinh, H. Cambon, M. Levasseur, and J. C. Baron, "Effects of capsular or thalamic stroke on metabolism in the cortex and cerebellum: A Positron Tomography Study," Stroke, vol. 21, no. 4, pp. 519-524, 1990.

[8] Y. Sakashita, H. Matsuda, K. Kakuda, and M. Takamori, "Hypoperfusion and vasoreactivity in the thalamus and cerebellum after stroke," Stroke, vol. 24, no. 1, pp. 84-87, 1993.

[9] H. Yamauchi, H. Fukuyama, Y. Nagahama, H. Okazawa, and J. Konishi, "A decrease in regional cerebral blood volume and hematocrit in crossed cerebellar diaschisis," Stroke, vol. 30, no. 7, pp. 1429-1431, 1999.

[10] M. Kraemer, T. Schormann, G. Hagemann, B. Qi, O. W. Witte, and R. J. Seitz, "Delayed shrinkage of the brain after ischemic stroke: preliminary observations with voxel-guided morphometry," Journal of Neuroimaging, vol. 14, no. 3, pp. 265272, 2004.

[11] J. Kim, S.-K. Lee, J. D. Lee, Y. W. Kim, and D. I. Kim, "Decreased fractional anisotropy of middle cerebellar peduncle in crossed cerebellar diaschisis: diffusion-tensor imagingpositron-emission tomography correlation study," American Journal of Neuroradiology, vol. 26, no. 9, pp. 2224-2228, 2005.

[12] C. Yu, C. Zhu, Y. Zhang et al., "A longitudinal diffusion tensor imaging study on Wallerian degeneration of corticospinal tract after motor pathway stroke," NeuroImage, vol. 47, no. 2, pp. 451$458,2009$.

[13] C. Weiller, F. Chollet, K. J. Friston, R. J. S. Wise, and R. S. J. Frackowiak, "Functional reorganization of the brain in recovery from striatocapsular infarction in man," Annals of Neurology, vol. 31, no. 5, pp. 463-472, 1992.

[14] B. Weder, U. Knorr, H. Herzog et al., "Tactile exploration of shape after subcortical ischaemic infarction studied with PET," Brain, vol. 117, no. 3, pp. 593-605, 1994.

[15] N. S. Ward, M. M. Brown, A. J. Thompson, and R. S. J. Frackowiak, "Neural correlates of motor recovery after stroke: A Longitudinal fMRI Study," Brain, vol. 126, no. 11, pp. 24762496, 2003.

[16] L. Wang, C. Yu, H. Chen et al., "Dynamic functional reorganization of the motor execution network after stroke," Brain, vol. 133, no. 4, pp. 1224-1238, 2010. 
[17] H. Baillieux, H. J. D. Smet, P. F. Paquier, P. P. De Deyn, and P. Mariën, "Cerebellar neurocognition: insights into the bottom of the brain," Clinical Neurology and Neurosurgery, vol. 110, no. 8, pp. 763-773, 2008.

[18] J. E. Desmond, J. D. E. Gabrieli, and G. H. Glover, "Dissociation of frontal and cerebellar activity in a cognitive task: evidence for a distinction between selection and search," NeuroImage, vol. 7, no. 4, part 1, pp. 368-376, 1998.

[19] P. Hubrich-Ungureanu, N. Kaemmerer, F. A. Henn, and D. F. Braus, "Lateralized organization of the cerebellum in a silent verbal fluency task: a functional magnetic resonance imaging study in healthy volunteers," Neuroscience Letters, vol. 319, no. 2, pp. 91-94, 2002.

[20] M. E. Raichle, J. A. Fiez, T. O. Videen et al., "Practice-related changes in human brain functional anatomy during nonmotor learning," Cerebral Cortex, vol. 4, no. 1, pp. 8-26, 1994.

[21] J. D. Schmahmann, "From movement to thought: anatomic substrates of the cerebellar contribution to cognitive processing," Human Brain Maping, vol. 4, no. 3, pp. 174-198, 1996.

[22] J. D. Schmahmann and J. C. Sherman, "The cerebellar cognitive affective syndrome," Brain, vol. 121, no. 4, pp. 561-579, 1998.

[23] H. Xiang, C. Lin, X. Ma et al., "Involvement of the cerebellum in semantic discrimination: An fMRI Study," Human Brain Mapping, vol. 18, no. 3, pp. 208-214, 2003.

[24] L. Sang, W. Qin, Y. Liu et al., "Resting-state functional connectivity of the vermal and hemispheric subregions of the cerebellum with both the cerebral cortical networks and subcortical structures," NeuroImage, vol. 61, no. 4, pp. 1213-1225, 2012.

[25] C. Habas, N. Kamdar, D. Nguyen et al., "Distinct cerebellar contributions to intrinsic connectivity networks," Journal of Neuroscience, vol. 29, no. 26, pp. 8586-8594, 2009.

[26] J. D. Schmahmann, "An emerging concept: the cerebellar contribution to higher function," Archives of Neurology, vol. 48, no. 11, pp. 1178-1187, 1991.

[27] C. J. Stoodley and J. D. Schmahmann, "Functional topography in the human cerebellum: a meta-analysis of neuroimaging studies," NeuroImage, vol. 44, no. 2, pp. 489-501, 2009.

[28] C. J. Stoodley and J. D. Schmahmann, "Evidence for topographic organization in the cerebellum of motor control versus cognitive and affective processing," Cortex, vol. 46, no. 7, pp. 831-844, 2010.

[29] J. A. Hosp and A. R. Luft, "Cortical plasticity during motor learning and recovery after ischemic stroke," Neural Plasticity, vol. 2011, Article ID 871296, 9 pages, 2011.

[30] J. W. Krakauer, "Motor learning: its relevance to stroke recovery and neurorehabilitation," Current Opinion in Neurology, vol. 19, no. 1, pp. 84-90, 2006.

[31] L. Shmuelof and J. W. Krakauer, "Are we ready for a natural history of motor learning?" Neuron, vol. 72, no. 3, pp. 469-476, 2011.

[32] J. Diedrichsen, J. H. Balsters, J. Flavell, E. Cussans, and N. Ramnani, "A probabilistic MR atlas of the human cerebellum," NeuroImage, vol. 46, no. 1, pp. 39-46, 2009.

[33] K. Murphy, R. M. Birn, D. A. Handwerker, T. B. Jones, and P. A. Bandettini, "The impact of global signal regression on resting state correlations: are anti-correlated networks introduced?" NeuroImage, vol. 44, no. 3, pp. 893-905, 2009.

[34] A. Weissenbacher, C. Kasess, F. Gerstl, R. Lanzenberger, E. Moser, and C. Windischberger, "Correlations and anticorrelations in resting-state functional connectivity MRI: a quantitative comparison of preprocessing strategies," NeuroImage, vol. 47, no. 4, pp. 1408-1416, 2009.
[35] M. Hampson, N. Driesen, J. K. Roth, J. C. Gore, and R. T. Constable, "Functional connectivity between task-positive and task-negative brain areas and its relation to working memory performance," Magnetic Resonance Imaging, vol. 28, no. 8, pp. 1051-1057, 2010.

[36] R. L. Buckner, F. M. Krienen, A. Castellanos, J. C. Diaz, and B. T. Yeo, "The organization of the human cerebellum estimated by intrinsic functional connectivity," Journal of Neurophysiology, vol. 106, no. 5, pp. 2322-2345, 2011.

[37] F. M. Krienen and R. L. Buckner, "Segregated fronto-cerebellar circuits revealed by intrinsic functional connectivity," Cerebral Cortex, vol. 19, no. 10, pp. 2485-2497, 2009.

[38] J. X. O’Reilly, C. F. Beckmann, V. Tomassini, N. Ramnani, and H. Johansen-Berg, "Distinct and overlapping functional zones in the cerebellum defined by resting state functional connectivity," Cerebral Cortex, vol. 20, no. 4, pp. 953-965, 2010.

[39] R. M. Kelly and P. L. Strick, "Cerebellar loops with motor cortex and prefrontal cortex of a nonhuman primate," Journal of Neuroscience, vol. 23, no. 23, pp. 8432-8444, 2003.

[40] S. H. A. Chen and J. E. Desmond, "Cerebrocerebellar networks during articulatory rehearsal and verbal working memory tasks," NeuroImage, vol. 24, no. 2, pp. 332-338, 2005.

[41] S. H. A. Chen and J. E. Desmond, "Temporal dynamics of cerebro-cerebellar network recruitment during a cognitive task," Neuropsychologia, vol. 43, no. 9, pp. 1227-1237, 2005.

[42] D. A. Gusnard and M. E. Raichle, "Searching for a baseline: functional imaging and the resting human brain," Nature Reviews Neuroscience, vol. 2, no. 10, pp. 685-694, 2001.

[43] S. G. Horovitz, A. R. Braun, W. S. Carr et al., "Decoupling of the brain's default mode network during deep sleep," Proceedings of the National Academy of Sciences of the United States of America, vol. 106, no. 27, pp. 11376-11381, 2009.

[44] K. Christoff, A. M. Gordon, J. Smallwood, R. Smith, and J. W. Schooler, "Experience sampling during fMRI reveals default network and executive system contributions to mind wandering," Proceedings of the National Academy of Sciences of the United States of America, vol. 106, no. 21, pp. 8719-8724, 2009.

[45] M. D. Greicius, B. Krasnow, A. L. Reiss, and V. Menon, "Functional connectivity in the resting brain: a network analysis of the default mode hypothesis," Proceedings of the National Academy of Sciences of the United States of America, vol. 100, no. 1, pp. 253-258, 2003.

[46] N. Filippini, B. J. MacIntosh, M. G. Hough et al., "Distinct patterns of brain activity in young carriers of the APOE- $\varepsilon 4$ allele," Proceedings of the National Academy of Sciences of the United States of America, vol. 106, no. 17, pp. 7209-7214, 2009.

[47] M. A. Foulkes, P. A. Wolf, T. R. Price, J. P. Mohr, and D. B. Hier, "The Stroke Data Bank: design, methods, and baseline characteristics," Stroke, vol. 19, no. 5, pp. 547-554, 1988.

[48] M. D. Fox, A. Z. Snyder, J. L. Vincent, M. Corbetta, D. C. Van Essen, and M. E. Raichle, "The human brain is intrinsically organized into dynamic, anticorrelated functional networks," Proceedings of the National Academy of Sciences of the United States of America, vol. 102, no. 27, pp. 9673-9678, 2005.

[49] D. Sridharan, D. J. Levitin, and V. Menon, "A critical role for the right fronto-insular cortex in switching between centralexecutive and default-mode networks," Proceedings of the National Academy of Sciences of the United States of America, vol. 105, no. 34, pp. 12569-12574, 2008. 
[50] C. M. Cirstea, A. Ptito, and M. F. Levin, "Feedback and cognition in arm motor skill reacquisition after stroke," Stroke, vol. 37, no. 5, pp. 1237-1242, 2006.

[51] N. C. Foley, R. W. Teasell, S. K. Bhogal, and M. R. Speechley, "Stroke rehabilitation evidence-based review: methodology", Topics in Stroke Rehabilitation, vol. 10, no. 1, pp. 1-7, 2003.

[52] A. W. S. Leung, S. K. W. Cheng, A. K. Y. Mak, K.-K. Leung, L. S. W. Li, and T. M. C. Lee, "Functional gain in hemorrhagic stroke patients is predicted by functional level and cognitive abilities measured at hospital admission," NeuroRehabilitation, vol. 27, no. 4, pp. 351-358, 2010.

[53] K. Oneş, E. Y. Yalçinkaya, B. C. Toklu, and N. Cağlar, "Effects of age, gender, and cognitive, functional and motor status on functional outcomes of stroke rehabilitation," NeuroRehabilitation, vol. 25, no. 4, pp. 241-249, 2009.

[54] S. E. McEwen, M. P. J. Huijbregts, J. D. Ryan, and H. J. Polatajko, "Cognitive strategy use to enhance motor skill acquisition poststroke: a critical review," Brain Injury, vol. 23, no. 4, pp. 263-277, 2009.

[55] F. Fan, C. Zhu, H. Chen et al., "Dynamic brain structural changes after left hemisphere subcortical stroke," Human Brain Mapping, 2012.

[56] L. V. Gauthier, E. Taub, C. Perkins, M. Ortmann, V. W. Mark, and G. Uswatte, "Remodeling the brain: plastic structural brain changes produced by different motor therapies after stroke," Stroke, vol. 39, no. 5, pp. 1520-1525, 2008.

[57] R. F. Gottesman and A. E. Hillis, "Predictors and assessment of cognitive dysfunction resulting from ischaemic stroke," The Lancet Neurology, vol. 9, no. 9, pp. 895-905, 2010.

[58] G. T. Stebbins, D. L. Nyenhuis, C. Wang et al., "Gray matter atrophy in patients with ischemic stroke with cognitive impairment," Stroke, vol. 39, no. 3, pp. 785-793, 2008.

[59] S. Lassalle-Lagadec, I. Sibon, B. Dilharreguy, P. Renou, O. Fleury, and M. Allard, "Subacute default mode network dysfunction in the prediction of post-stroke depression severity," Radiology, vol. 264, no. 1, pp. 218-224, 2012.

[60] E. M. Nomura, C. Gratton, R. M. Visser, A. Kayser, F. Perez, and M. D’Esposito, "Double dissociation of two cognitive control networks in patients with focal brain lesions," Proceedings of the National Academy of Sciences of the United States of America, vol. 107, no. 26, pp. 12017-12022, 2010. 

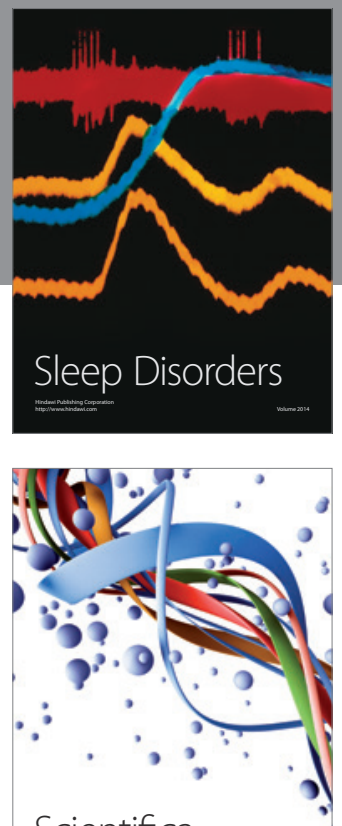

Scientifica
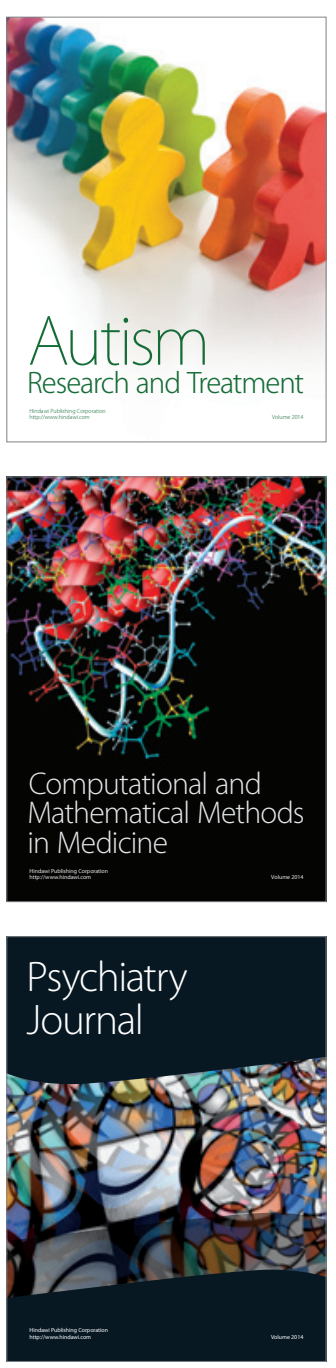
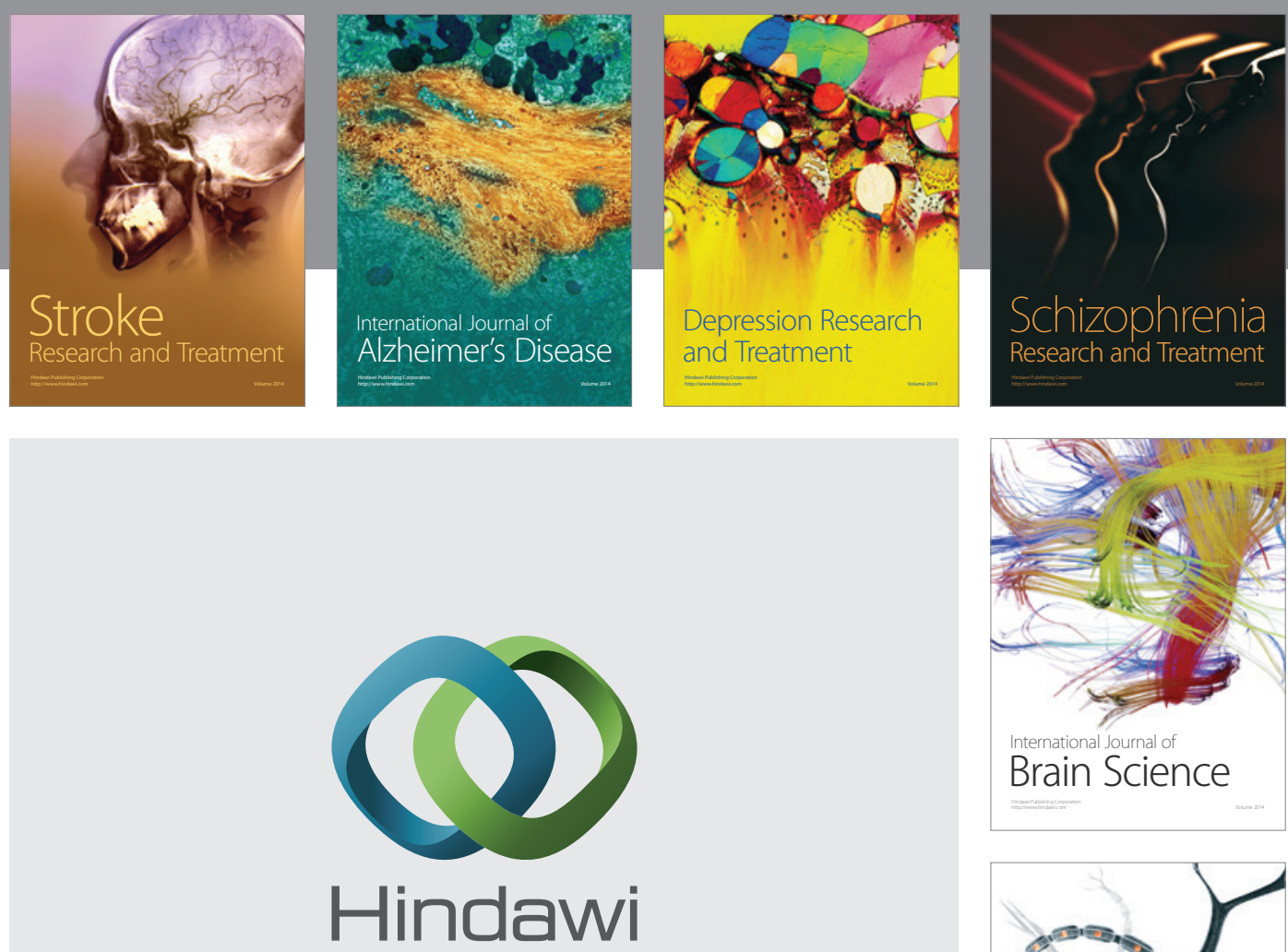

Submit your manuscripts at

http://www.hindawi.com
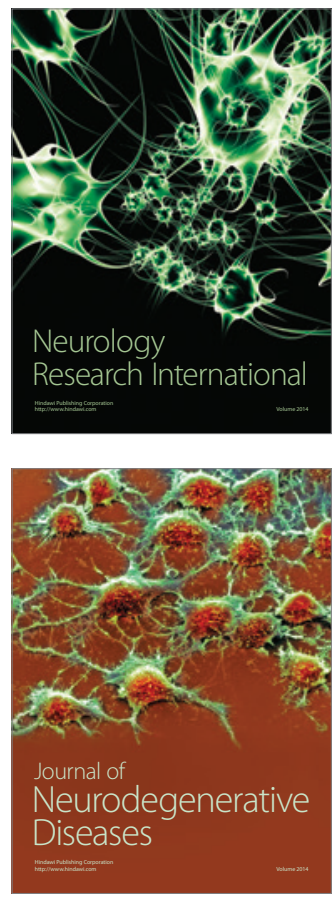

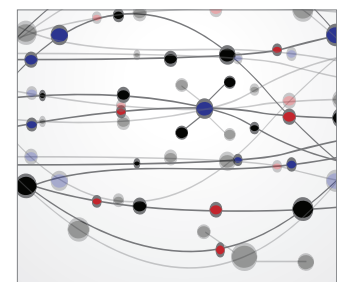

The Scientific World Journal
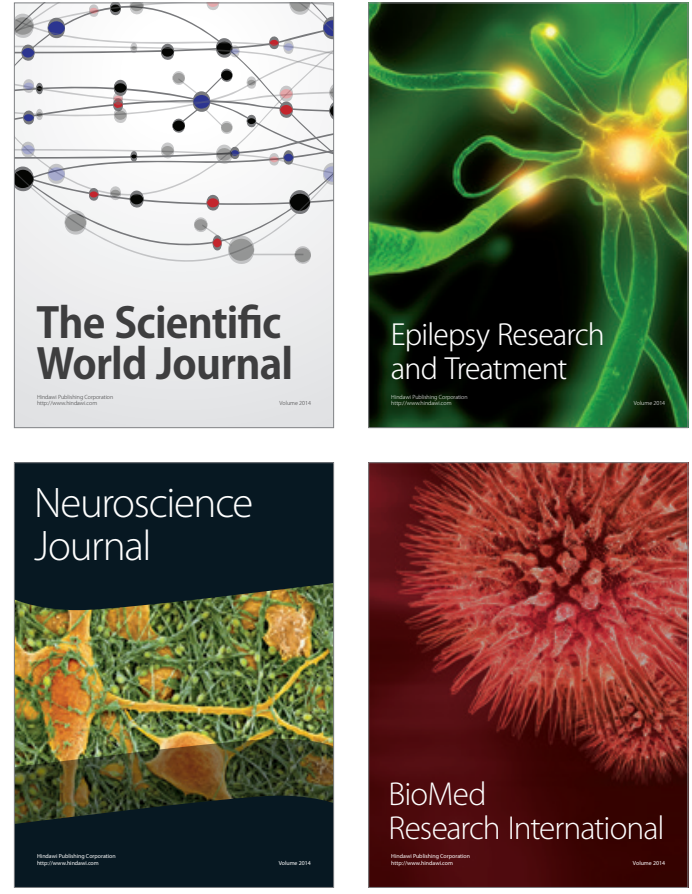

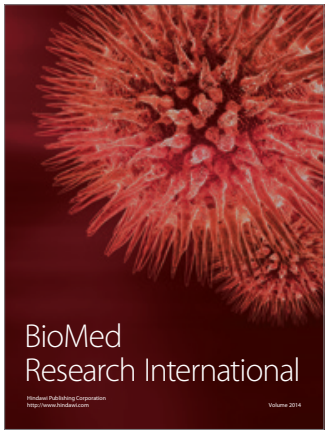

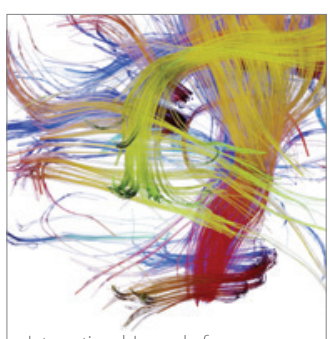

Brain Science

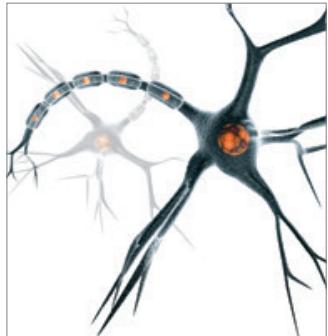

Neural Plasticity
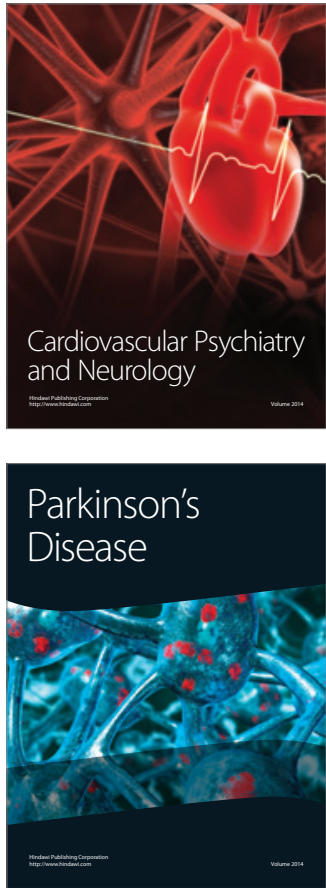